Supplement of Wind Energ. Sci., 5, 745-758, 2020

https://doi.org/10.5194/wes-5-745-2020-supplement

(C) Author(s) 2020. This work is distributed under

the Creative Commons Attribution 4.0 License.

(c) (1)

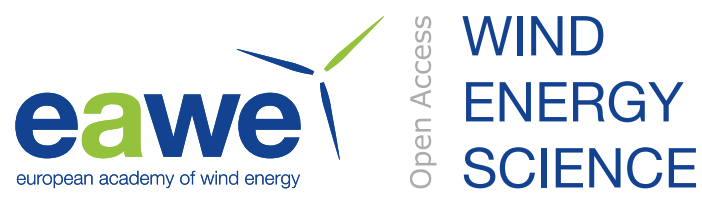

Supplement of

\title{
Experimental investigation of aerodynamic characteristics of bat carcasses after collision with a wind turbine
}

\author{
Shivendra Prakash and Corey D. Markfort \\ Correspondence to: Corey D. Markfort (corey-markfort@uiowa.edu)
}

The copyright of individual parts of the supplement might differ from the CC BY 4.0 License. 
This supplementary information document presents the detailed procedure and results of Gaussian distribution fitting to pixel coordinate and intensity measurements of Hoary bat drop \#1 experiment images, to determine the carcass centroid (Section S1). The document describes the steps of the proposed methodology to determine carcass drag coefficient $\left(C_{d}\right)$, by optimized coarsening window $\left(\Delta t_{c}\right)$ and initial drop height $\left(z_{0}\right)$ (Section S2). It also presents the multivariable optimization procedure illustrated in figures showing the filtering window $(\Delta t)$ vs. root mean square error in velocity $\left(R M S E_{w}\right)$ and $R M S E_{w}$ heatmap in $z_{0}-C_{d}$ plane, for each of the three bat species (Section S3).

\section{S1. Determination of position from images using recommended method in Mann et al. (1999)}

The proposed methodology determines the position on images with the precision of 0.10 pixels -0.02 pixels, by fitting a Gaussian function to the particle pixel grey intensity. We show here results from the recommended procedure as applied to the position measurements of drop \#1 of Hoary bat carcass experiment. The carcass position measurements from the recommended method is compared with the earlier measurements obtained from mean of carcass top and bottom pixel coordinates. Figure S1 shows the snapshot of the greyscale image at $t=0.420 \mathrm{~s}$ during drop \#1 of Hoary bat carcass. The image has been cropped to focus on the region around the carcass image. The greyscale intensity between $0-255$ is described at each pixel coordinate encompassed in Fig. S1. The rectangular area enclosing the carcass and surrounding interrogation region covers 15 pixels (horizontally) and 13 pixels (vertically). The carcass image occupies eight pixels or $57 \mathrm{~mm}(1$ pixel $=7.10 \mathrm{~mm})$. The coordinates of each pixel in horizontal $\left(x_{\text {pixel }}\right)$ and vertical $\left(z_{\text {pixel }}\right)$ direction and pixel intensities $\left(I_{\text {pixel }}\right)$ displayed in Fig. S1 were recoded. The pixel intensities were summed over all $x_{\text {pixels }}$ to yield the total pixel intensity $\left(I_{\text {Total }}\right)$ as a function of $z_{\text {pixel }}$. Table S1 shows $I_{\text {Total }}$ at each $z_{\text {pixel }}$ of the rectangular area in Fig. S1.

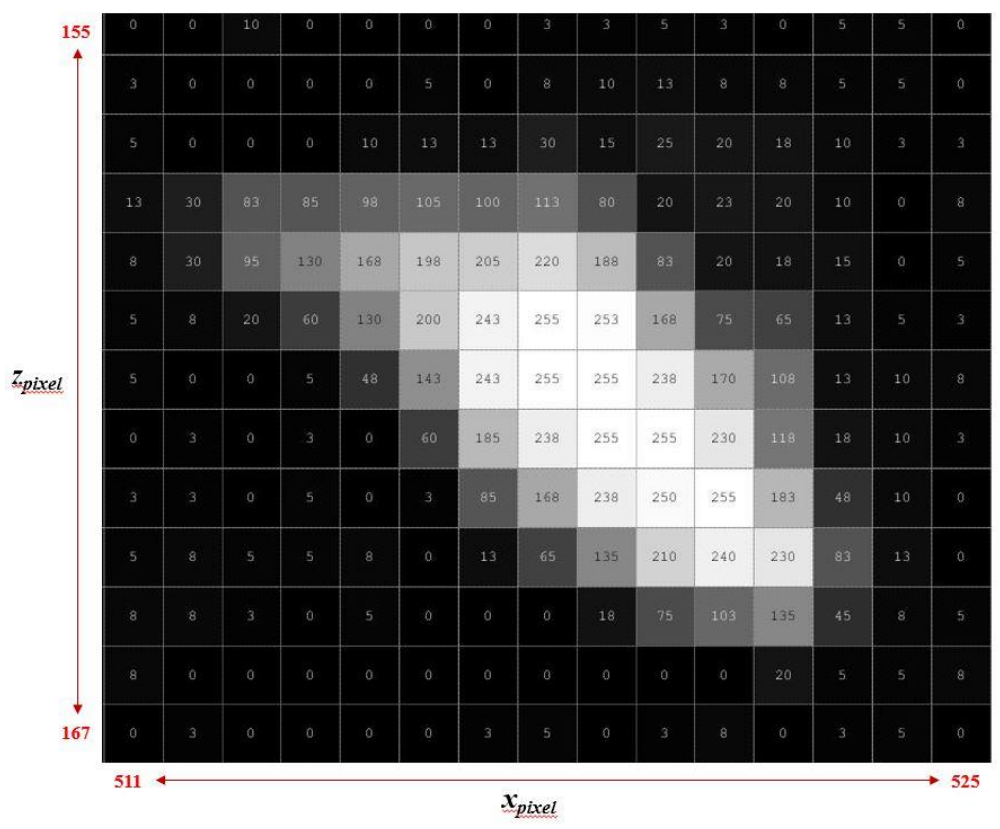

Fig. S1: Selected grey area pixels around carcass at $t=0.420 \mathrm{~s}$ 
Table S1: $I_{\text {Total }}$ at a given $z_{p i x e l}$

\begin{tabular}{|c|c|}
\hline$z$ pixel & ITotal \\
\hline 155 & 34 \\
\hline 156 & 65 \\
\hline 157 & 165 \\
\hline 158 & 780 \\
\hline 159 & 1383 \\
\hline 160 & 1503 \\
\hline 161 & 1501 \\
\hline 162 & 1378 \\
\hline 163 & 1251 \\
\hline 164 & 1020 \\
\hline 165 & 413 \\
\hline 166 & 46 \\
\hline 167 & 30 \\
\hline
\end{tabular}

Figure S2 shows $z_{\text {pixel. }}$ vs. $I_{\text {Total }}$ plot (red dots) for the measurements in Table S1. The centroid of greyscale intensity in Table S1 was computed by averaging each $z_{\text {pixel }}$ based on its $I_{\text {Total }}$ value (Eq. (S1)) and was found to be 161.123. The carcass centroid from top and bottom pixel coordinate was estimated as 160 . This procedure was repeated for next four consecutive images and carcass centroid was calculated for each of the image frames.

$$
z_{c, \text { new }}=\frac{\sum I_{\text {pixel }} Z_{\text {pixel }}}{\sum I_{\text {pixel }}}
$$

Table S2 below displays the comparison of $z_{c}$ from two methods, i.e., mean top and bottom method ( $2^{\text {nd }}$ column) and center of intensity method ( $3^{\text {rd }}$ column) for the selected greyscale frames. It is evident from the table that maximum $z_{c}$ difference is of the order of approximately 1 pixel from the two methods.

Table S2: Comparison of $z c$

\begin{tabular}{|c|c|c|c|}
\hline$t(\mathbf{s})$ & $\begin{array}{c}z_{c} \text { (Mean top and bottom method) } \\
(\mathbf{m})\end{array}$ & $\begin{array}{c}z_{c}(\text { Center of Intensity method, S1) } \\
(\mathbf{m})\end{array}$ & $\left|\Delta z_{c}\right|(\mathbf{m m})$ \\
\hline 0.420 & 5.928 & 5.919 & 9 \\
\hline 0.424 & 5.903 & 5.900 & 3 \\
\hline 0.428 & 5.879 & 5.876 & 3 \\
\hline 0.432 & 5.857 & 5.853 & 4 \\
\hline 0.436 & 5.836 & 5.830 & 6 \\
\hline
\end{tabular}

Mann et al. (1999) proposed fitting particle images with a Gaussian shape function to determine the position from the images. A similar procedure was applied to the measured distribution shown in Fig. S2 to compute the centroid of the distribution, $z_{c}$. The Gaussian distribution expressed by Eq. (S2) was fitted to $z_{p i x e l}$ vs. I Itotal data in Table $\mathrm{S} 1$ to find the best estimate of carcass centroid, $z_{c}$, at $t=0.420 \mathrm{~s}$. Figure S2 shows the Gaussian distribution (black line) fitted to $z_{p i x e l}$ vs. ITotal measurements to give $z_{c}=160.988$. 


$$
I_{\text {Total }}=\frac{1}{\sigma_{z} \sqrt{2 \pi}} e^{-\frac{1}{2}\left(\frac{z_{\text {pixel }}-z_{c}}{\sigma_{z}}\right)^{2}}
$$

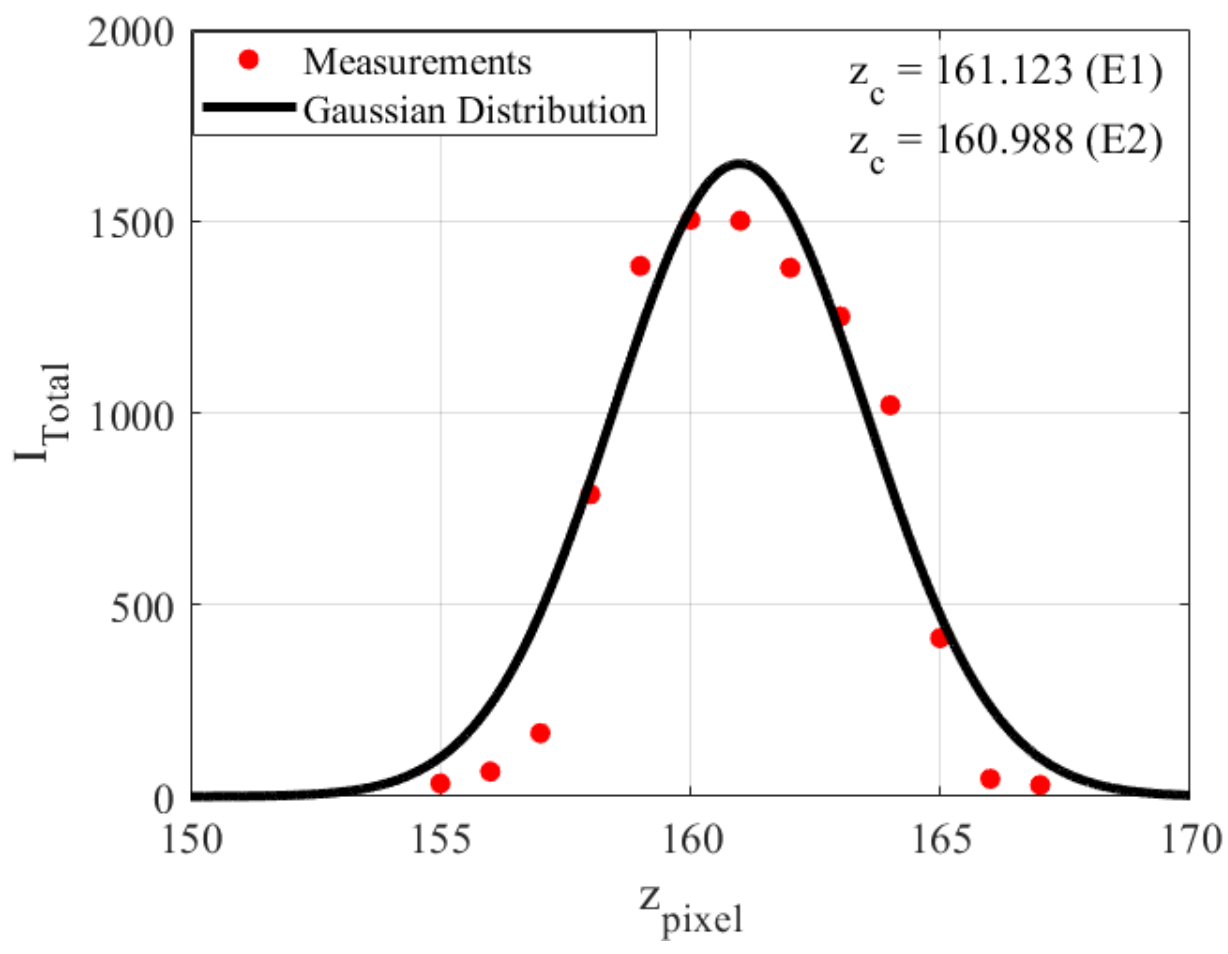

Fig. S2: Gaussian distribution fitted to $z_{\text {pixel }}$ vs. I Iotal $_{\text {measurements }}$

The Gaussian distribution was fitted to zpixel vs. ITotal measurements of the next consecutive images to determine the respective centroids. Table $\mathrm{S} 3$ shows the comparison of the carcass centroid estimated using top and bottom pixels ( $2^{\text {nd }}$ column $)$ and by fitting Gaussian distribution ( $3^{\text {rd }}$ column) to $z_{\text {pixel }}$ vs. $I_{\text {Total }}$ measurements. Again, we notice from Table S3 that the difference in the $z_{c}$ estimates from the two approaches are of the order of 1 pixel.

Table S3: Comparison of $z c$

\begin{tabular}{|c|c|c|c|}
\hline $\boldsymbol{t}(\mathbf{s})$ & $z_{c}$ (Mean top and bottom method) & $\begin{array}{c}z_{c} \text { (Gaussian fitting, S2) } \\
(\mathbf{m})\end{array}$ & $\left|\Delta z_{c}\right|(\mathbf{m m})$ \\
\hline 0.420 & 5.928 & 5.920 & 8 \\
\hline 0.424 & 5.903 & 5.898 & 5 \\
\hline 0.428 & 5.879 & 5.875 & 4 \\
\hline 0.432 & 5.857 & 5.852 & 5 \\
\hline 0.436 & 5.836 & 5.829 & 7 \\
\hline
\end{tabular}




\section{S2. Proposed multivariable optimization algorithm}

1. With the assumption, $w_{0}=0$, one degree of freedom in the problem is reduced, leaving three degrees of freedom $\left(\Delta t_{c}, z_{0}, C_{d}\right)$.

2. An array of plausible $z_{0}$ and $C_{d}$ values is declared and then the ballistics model was solved for the prescribed values of $z_{0}$ and $C_{d}$. The $z_{0}$ array with $m$ elements and $C_{\mathrm{d}}$ array with $n$ elements results in $m \times n$ fall trajectories.

3. An array representing different resolutions, $\Delta t$, of measured velocity with $p$ elements is defined. The measured velocity at different resolutions is fitted to $m \times n$ fall trajectories. This leads to $m \times n$ number of $z_{0}-C_{d}$ combinations of fall trajectories fitted to $p$ different resolutions of measured velocity.

4. For each of the $m \times n \times p$ fitting events, root mean square error in fall velocity $\left(R M S E_{w}\right)$ at $i^{\text {th }}$ time instant is estimated using the following formula:

$$
R M S E_{w}=\sqrt{\frac{\sum_{i=1}^{n}\left(w_{\text {model }}(i)-w_{\text {field }}(i)\right)^{2}}{n}}
$$

where $w_{\text {model }}$ represents the velocity obtained from the ballistics model solution and $w_{\text {field }}$ represents the measured velocity from high - speed imaging of carcass drop experiments. The variable $n$ is the number of data points in velocity time series for a specific coarsening window.

5. For $m \times n$ combinations of $z_{0}$ and $C_{d}, \Delta t$ vs. $R M S E_{w}$ is plotted. The range of $\Delta t$ values over which $R M S E_{w}$ remains invariant for $z_{0}-C_{d}$ combinations is identified. The basis of selecting $\Delta t$ range of invariant $R M S E_{w}$ is: the relative error in successive $R M S E_{w}$ values being less than $10 \%$.

$$
\operatorname{RELError}(i)=\left(\frac{R M S E_{w}(i+1)-R M S E_{w}(i)}{R M S E_{w}(i)}\right) \times 100
$$

where $R M S E_{w}(i+1)$ and $R M S E_{w}(i)$ are the two successive values in $R M S E_{w}$ vector at $(i+1)^{\text {th }}$ and $i^{\text {th }}$ time step for a specific $z_{0}-C_{\mathrm{d}}$ combination. RELError $(i)$ is the relative error in $R M S E_{w}$ at $i^{\text {th }}$ time step.

6. For each element in $\Delta t$ vector (which corresponds to invariant $R M S E_{w}$ ) representing a plausible optimum resolution of the measured velocity, the optimal $z_{0}$ and $C_{d}$ is computed by defining $\left(R M S E_{w}\right)_{\min }$ as the objective function. The temporal resolution of extracted data $(0.004 \mathrm{~s})$ yields serious scatter in the measured velocity which makes it impossible to find the best - fit of ballistics model to the measured velocity. For large filtering window of the measured data, the order of the ballistics model becomes equal to the number of the data points; hence giving the biased estimate of $R M S E_{w}$. The objective for selecting the $\Delta t$ range of constant $R M S E_{w}$ is to find an unbiased estimator of the goodness of fit and therefore, range of $\Delta t$ yielding invariant $R M S E_{w}$ is selected as possible candidates for determining the optimal coarsening window.

7. From step 6, a pool of initial positions $\left(z_{0}\right)$ and drag coefficients $\left(C_{d}\right)$ for varying resolutions of measured velocity (embedded in $\Delta t$ vector corresponding to constant $\left.\left(R M S E_{w}\right)_{\min }\right)$ is obtained. Out of this pool, the value of $\Delta t, z_{0}$ 
and $C_{d}$ giving the global minimum $\left(R M S E_{w}\right)_{\min }$ is selected as optimum data resolution, initial position, and carcass drag coefficient, respectively.

8. To test the accuracy of the optimum $\Delta t_{c}, z_{0}$ and $C_{d}$, the analytical solution of the ballistics model from optimized $z_{0}$ and $C_{d}$ estimates is compared with the measured position and velocity at the optimum resolution $\left(\Delta t_{c}\right)$.

\section{S3. Optimized drag coefficient estimates}

The optimization algorithm steps described in section S2 were applied to the carcass fall velocity data to determine the carcass drag coefficient of the three discovered species. Following are the results of the optimization process:

\section{S3.1 Hoary bat}

For carcass drop experiment, $z o$ was defined between $7.40 \mathrm{~m}$ and $7.80 \mathrm{~m}$ at an increment of $0.01 \mathrm{~m}$ whereas $C_{d}$ array was selected between 0.50 and 1 with differential $C_{d}$ being 0.01 . In this manner, there are overall $41 \times 51 z_{0}-C_{d}$ combinations leading to equal number of carcass fall trajectories. The $\Delta t$ array was chosen from $0.004 \mathrm{~s}$ to $0.512 \mathrm{~s}$ at an increment of $0.004 \mathrm{~s}$. This declaration of $z_{0}-C_{d}-\Delta t$ culminated in $41 \times 51 \times 128$ ballistics model fitting events to the measured velocity. For each of these cases, $R M S E_{w}$ was calculated through difference in the modelled velocity and measured velocity values.

Three - point centered moving average of $R M S E_{w}$ vector was computed and plotted with $\Delta t$ (for all $z_{0}-C_{d}$ combinations) in order to identify $\Delta t$ range of invariant $R M S E_{w}$ on the basis of RELError $<10 \%$. Figure S3 (a) demonstrates the $\Delta t$ vs. $R M S E_{w}$ plot, for the lower $\left(z_{0}=7.40 \mathrm{~m}\right.$ and $\left.C_{d}=0.50\right)$ and upper $\left(z_{0}=7.80 \mathrm{~m}\right.$ and $\left.C_{d}=1\right)$ bounds of $z_{0}$ and $C_{d}$ array respectively. The $\Delta t$ range corresponding to invariant $R M S E_{w}$, was found to be between 0.060 and $0.104 \mathrm{~s}$ (region between the two vertical arrows in Fig. S3 (a)). Optimum $z_{0}$ and $C_{d}$ were calculated by minimizing $R M S E_{w}$ for each element in $\Delta t$ vector corresponding to invariant $R M S E_{w}$. Ultimately, the global minimum $\left(R M S E_{w}\right)_{\min }$ was selected as a criterion for $\Delta t_{c}, z_{0}$ and $C_{d}$, in highlighted spectrum of $\Delta t$ giving invariant $R M S E_{w}$. Fig. $\mathrm{S} 3$ (b) shows the plot of $R M S E_{w}$ in the $z_{0}-C_{d}$ plane at an optimal resolution of $\Delta t_{c}=0.104 \mathrm{~s}$, in highlighted domain of $\Delta t$ vector. The red dot in the heatmap displays the optimum $z_{0}=7.58 \mathrm{~m}$ and $C_{d}=0.70$ yielding global minimum $\left(R M S E_{w}\right)_{\min }$ of $0.067 \mathrm{~m} / \mathrm{s}$. 


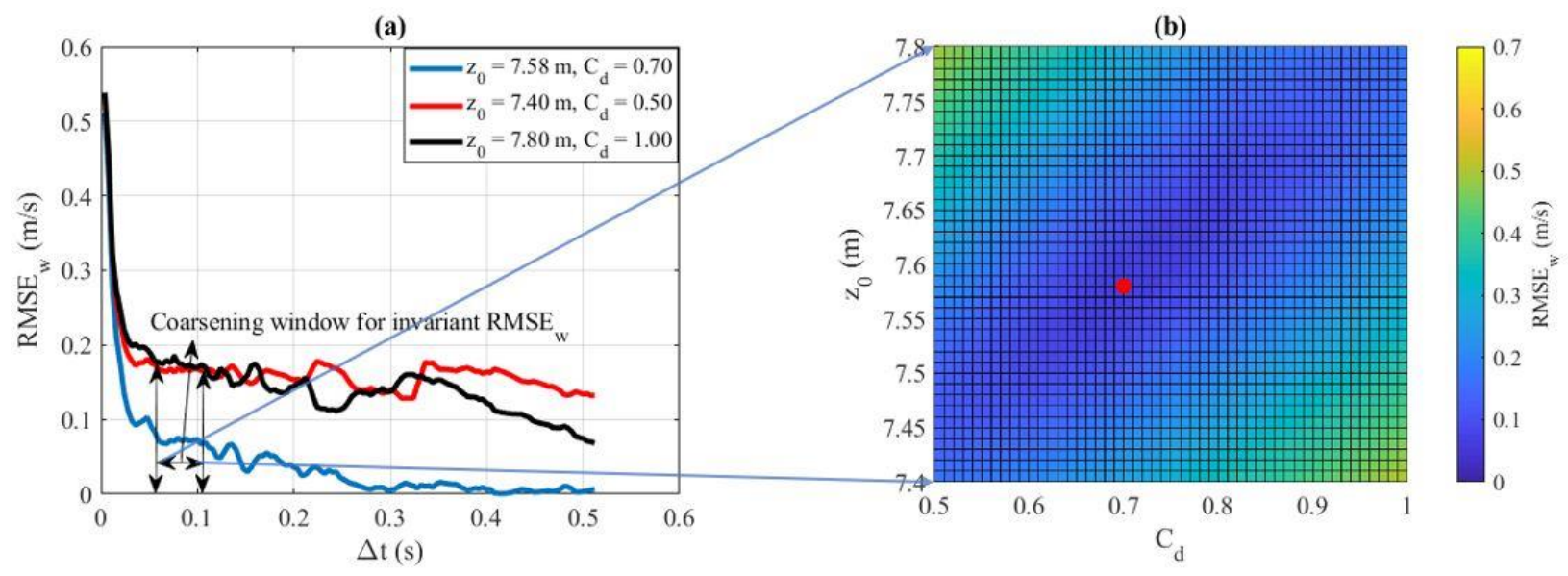

Fig. S3: (a) $\Delta t$ vs. $R M S E_{w}$ plot; (b) $R M S E_{w}$ heatmap in $z_{0}-C_{d}$ plane (Hoary bat)

\section{S3.2 Eastern Red bat}

For analyzing Eastern Red bat, $z o, C_{d}$ and $\Delta t$ were defined in the same manner as with the Hoary bat. This declaration of $z_{0}-C_{d}-\Delta t$ culminated in $41 \times 51 \times 128$ ballistics model fitting events to the measured velocity. For each of these cases, $R M S E_{w}$ was calculated through difference in the modelled velocity and measured velocity values. Again, three - point centered moving average of $R M S E_{w}$ vector was computed and plotted with $\Delta t$ (for all $z_{0}-C_{d}$ combinations) in order to identify $\Delta t$ range of invariant $R M S E_{w}$ on the basis of RELError $<10 \%$.

Figure S4 (a) demonstrates the $\Delta t$ vs. $R M S E_{w}$ plot, for the lower and upper bounds of $z_{0}$ and $C_{d}$ array respectively. The spectrum of $\Delta t$ corresponding to invariant $R M S E_{w}$ was found to be between $0.080 \mathrm{~s}$ and $0.152 \mathrm{~s}$ (region between the two vertical arrows in Fig. S4 (a)). For each element in the above - mentioned $\Delta t$ range of invariant $R M S E_{w}$, the optimum $z_{0}$ and $C_{d}$ were calculated by minimizing $R M S E_{w}$ and then from this pool of $\left(R M S E_{w}\right)_{\text {min }}$, global minimum $\left(R M S E_{w}\right)_{\min }$ was selected as the criteria to identify $\Delta t_{c}$, optimized $z_{0}$ and $C_{d}$ for that specific carcass drop experiment. Figure S4 (b) shows the plot of $R M S E_{w}$ in $z_{0}-C_{d}$ plane, at an optimum filtering of $\Delta t_{c}=0.152 \mathrm{~s}$, in the marked range of $\Delta t$ vector. The optimized $z_{0}(7.63 \mathrm{~m})$ and $C_{d}(0.80)$ corresponding to the global minimum $\left(R M S E_{w}\right)_{\min }$ of $0.044 \mathrm{~m} / \mathrm{s}$, is highlighted by red dot in Fig. S4 (b). 


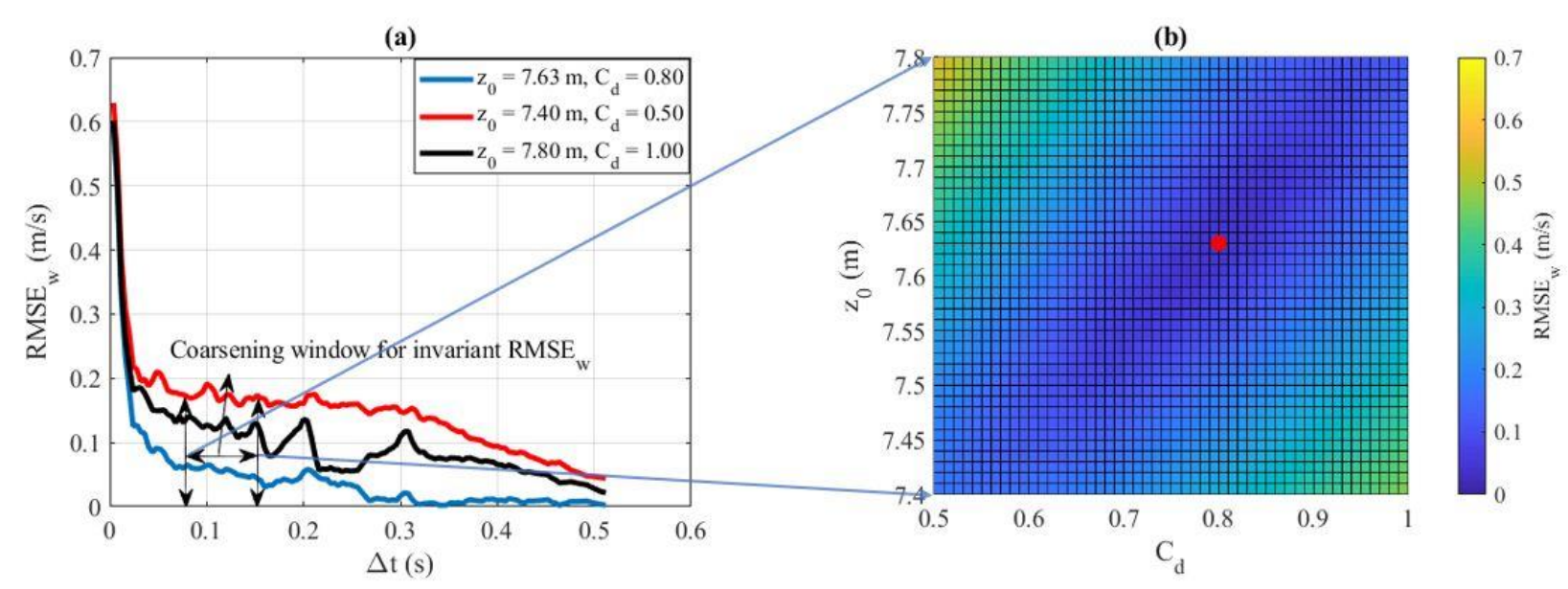

Fig. S4: (a) $\Delta t$ vs. $R M S E_{w}$ plot; (b) $R M S E_{w}$ heatmap in $z_{0}-C_{d}$ plane (Eastern Red bat)

\section{S3.3 Evening Bat}

For Evening bat, $z_{0}$ array was declared between $6.90 \mathrm{~m}$ and $7.70 \mathrm{~m}$ with differential $z_{0}$ being $0.01 \mathrm{~m}$ whereas $C_{d}$ vector was defined between 0.90 and 1.20 at an increment of 0.01. $\Delta t$ array was kept the same as it was with Eastern Red bat and Hoary bat. This declaration generated overall $81 \times 31 \times 128$ ballistics model fitting events to the different filtering windows of measured velocity.

Figure S5 (a) shows the $\Delta t$ vs. $R M S E_{w}$ (moving averaged) plot, for the lower and upper bounds of $z_{0}$ and $C_{d}$ array respectively. $\Delta t$ range corresponding to invariant $R M S E_{w}$ was established between $0.132 \mathrm{~s}$ and $0.144 \mathrm{~s}$ (region between vertical arrows in Fig. S5 (a)) and global minimum $\left(R M S E_{w}\right)_{\min }$ was selected as a criterion for $\Delta t_{c}$, optimized $z_{0}$ and $C_{d}$, within marked range of $\Delta t$. The red dot in Fig. S5 (b) represents optimal values of $z_{0}=7.20 \mathrm{~m}$ and $C_{d}=1.01$ with global minimum $\left(R M S E_{w}\right)_{\min }$ of $0.078 \mathrm{~m} / \mathrm{s}$ for $\Delta t_{c}=0.144 \mathrm{~s}$.

(a)

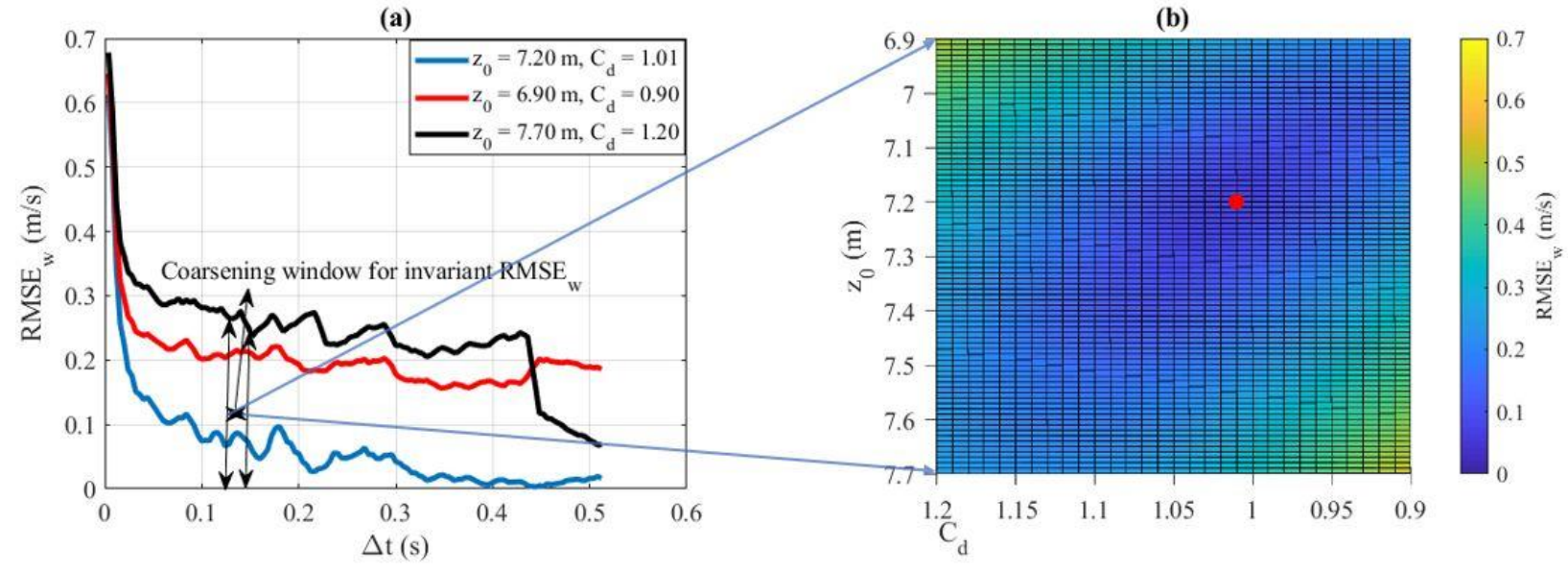

Fig. S5: (a) $\Delta t$ vs. $R M S E_{w}$ plot; (b) $R M S E_{w}$ heatmap in $z_{0}-C_{d}$ plane (Evening bat) 